\title{
Are there still indications for whole brain irradiation in 2021?
}

\author{
Karin Dieckmann $\cdot$ Harald Herrmann
}

Received: 7 April 2021 / Accepted: 10 April 2021 / Published online: 7 May 2021

(C) The Author(s) 2021

\begin{abstract}
Summary Brain metastases (BM) are the most frequent intracranial tumors in adults. About $10-20 \%$ of the patients with cancer will develop them. Historically, most of the patients with brain metastases were treated with whole brain radiotherapy (WBRT). The intention was to control the metastases and to eliminate distant micrometastases. Randomized control trials showed no difference in survival in patients with single and oligometastases treated with WBRT compared with stereotactic radiosurgery (SRS). To avoid treatment-related toxicities with neurocognitive decline, indications for WBRT are changing. High precision therapy with SRS or postoperative stereotactic treatments have become increasingly important. Only in exceptional cases is WBRT still the treatment of choice.
\end{abstract}

Keywords Brain metastasis - Neurocognitive impairment - Whole brain radiotherapy $\cdot$ Stereotactic radiotherapy

\section{Introduction}

The management of brain metastases (BM) is very complex. The performance status of the patient, local and distant control of the primary tumor and comorbidities have to be taken into account. Most frequently BMs are diagnosed in patients with lung cancer $(20-56 \%$ of all BMs), breast cancer $(5-20 \%)$ and melanoma (7-16\%) accounting for $70-80 \%$ of all brain metastases [9-11].

Based on magnetic resonance imaging (MRI) as the standard diagnostic tool, the number of newly diag-

K. Dieckmann $(\bowtie) \cdot$ H. Herrmann

Department of Radio-Oncology, Medical University of Vienna, Waehringer Guertel 18-20, 1090 Vienna, Austria karin.dieckmann@meduniwien.ac.at nosed symptomatic and asymptomatic BMs continues to increase. Historically, all patients with multiple BMs were treated with whole brain radiotherapy (WBRT). The goal of WBRT was symptom reduction and palliation, to stop brain metastases progression and possibly to prolong overall survival (OS). However, neurocognitive functional decline has been reported in $31-57 \%$ of the patients at 3 months and $48-89 \%$ at 1 year after WBRT [1].

In order to maintain neurocognitive function, stereotactic radiosurgery (SRS) and fractionated stereotactic radiosurgery (fSRS) have been implemented in the treatment of patients in recent years. This was not only performed in patients with a single metastasis, but also in patients with brain oligometastases, despite the higher risk of new intracranial relapses. Modern treatment techniques such as volumetric modulated arc therapy (VMAT) allow for WBRT with hippocampal-avoidance and dose-escalation with oligometastases [12, 14, 15]. In a randomized doubleblind, placebo-controlled phase III trial (RTOG 0614), the use of memantine, a neuroprotective compound, during and after WBRT has resulted in better cognitive function over time [24].

Traditional chemotherapy has played a limited role in the management of BM. However, recent advances in targeted therapy and checkpoint inhibitors have improved survival in patients with BM. Modern systemic treatments as tyrosine kinase inhibitors (TKI) and immunotherapies can cross the blood-brain barrier and have improved the results of systemic treatment in patients with asymptomatic disease [7].

In the modern era, therefore, a personalized treatment decision for patients with BMs has to be performed. To choose an adequate treatment, scores such as the graded prognostic assessment (GPA score) and the recursive partitioning analysis (RPA) including prognostic factors as histology of the primary tu- 
mor, the target mutation status, age of the patient, Karnofsky performance score (KPS), number and volume of the metastases with or without neurological symptoms, and the extra cranial tumor extension and activity are required [23].

\section{WBRT as first choice of treatment in patients with brain metastases from solid tumors}

WBRT was the standard treatment for most patients with BM. Today WBRT is indicated for patients with poor prognosis if BM are unsuitable for radiosurgery or surgery. According to the European Association of Neuro-Oncology (EANO) guidelines on brain metastases from solid tumors, WBRT in combination with corticosteroids is recommended in patients with (a) multiple brain metastases or presenting with (b) uncontrolled primary tumor or (c) multiple extracerebral metastases, if they are symptomatic [7]. Upfront WBRT remains a standard approach for patients with multiple brain metastases even though the Quality of Life after Treatment for Brain Metastases (QUARTZ) trial cannot assert the advantage of WBRT plus best supportive care compared with best supportive care alone in patients [19].

Median survival following WBRT alone ranges from 3 to 6 months, with $10-15 \%$ of patients alive at 1 year [8]. Various fractionation schedules may be used (30 Gy in 10 daily fractions, 20 Gy in 4 or 5 daily fractions, or others). None has proven superiority in terms of prolonging OS or better neurologic function or symptom control described in a meta-analysis of 39 trials by Tsao et al. [25].

\section{WBRT in patients with recurrent brain metastases after preliminary SRS}

Salvage WBRT can be an option in patients with favorable prognostic factors and recurrence of multiple new brain metastases relatively long time after SRS, who are not suitable for new SRS. No prospective studies are available, but salvage SRS after WBRT has been widely performed.

\section{WBRT in combination with SRS or surgery}

WBRT leads to an improved outcome in patients with single metastases, including improved overall survival, less development of new brain relapses and longer duration of functional independence [3-5]. Patients with brain oligometastases (1-4 metastases) showed no improvement with combined treatment of WBRT and SRS. Studies compared WBRT plus SRS with SRS alone [6]. Overall survival after combined treatment did not differ from SRS alone, only the distant brain metastases were more frequent in patients treated with SRS alone. Adjuvant WBRT following surgery increases local control and reduces distant relapses in patients with brain metastases $>3 \mathrm{~cm}$. Regardless which kind of combination is performed WBRT increases the risk of neurocognitive decline, transient lower physical functioning and more fatigue. Based on these results, the American Society for Radiation Oncology (ASTRO) does not recommend a combination of SRS and WBRT routinely in patients with limited BMs, but to perform frequent MRI surveillance.

\section{WBRT in patients with leptomeningeal carcinomatosis}

Palliative indications for WBRT are leptomeningeal carcinomatoses (LC) with multifocal spread of tumor cells and/or cerebrospinal fluid (EANO-ESMO guidelines) and eventually concomitant cerebral lesions. The incidence is approximately $5-10 \%$ of patients presenting with metastatic disease $[17,18]$. In these patients WBRT can delay neurologic deterioration without an increase in OS.

\section{WBRT as prophylactic cranial irradiation}

Prophylactic cranial irradiation (PCI) in small cell lung cancer (SCLC) is still the gold standard for patients with limited tumor progression or with very good treatment response and stable extracranial disease after chemotherapy. Auperin et al. in 1999 performed a meta-analysis of 7 studies comprising 987 patients. They found that PCI showed an improvement in the 3 -year OS of $5.4 \%$ (15.3\% to $20.7 \%$ ) [22]. The results of this meta-analysis could not be confirmed in a prospective MRI-based phase III study by Takahashi et al. [16]. New treatment concepts are under evaluation recommending close monitoring by MRI controls in compliant patients and local SRS in case of single or oligometastases.

\section{Patients with asymptomatic brain metastases}

Staging of the brain at the time of diagnosis is the gold standard for patients with solid tumors. Many clinically asymptomatic patients may have BMs detected by MRI. According to the recommendations of the EANO Guidelines [7], conventional chemotherapy may be the initial treatment for patients with BM from chemosensitive tumors like SCLC and breast cancer. Patients with brain metastases from NSCLC harboring activating EGFR mutations or ALK rearrangement can benefit from specific TKIs or patients with HER2-positive breast cancer can benefit from lapatinib alone or in combination with capecitabine. A benefit of ipilimumab or BRAF inhibitors on BM of melanomas has been frequently reported in the literature.

The response rate to targeted therapies and immunotherapies seems to be higher than those observed after chemotherapy. Currently new generations of small molecule inhibitors are under evaluation. In case of good response of asymptomatic BMs WBRT or 
SRS can be postponed or completely avoided. Prerequisite for this procedure is close MRI monitoring to detect growing BMs as soon as possible.

Systemic targeted therapies have become a more important part of BM treatment especially in patients with asymptomatic or oligosymptomatic disease.

\section{Conclusion}

Radiotherapy still plays a major role, as the activity of systemic chemotherapy within brain parenchyma remains limited [2]. WBRT does not prevent the development of new BMs and has a limited influence on overall survival. It can lead to decline of neurocognitive function and quality of life. Therefore, new local treatment options as SRS and fSRS have replaced WBRT in single and oligometastases. Only in case of multiple BMs unsuitable for SRS or surgery and in patients with poor prognosis, is WBRT the treatment of choice. New medical therapies passing the blood-brain barrier (BBB) may allow to postpone WBRT in asymptomatic patients and may inhibit the neurocognitive decline and maintain the quality-oflife.

Funding Open access funding provided by Medical University of Vienna.

Conflict of interest K. Dieckmann and H. Herrmann declare that they have no competing interests.

Open Access This article is licensed under a Creative Commons Attribution 4.0 International License, which permits use, sharing, adaptation, distribution and reproduction in any medium or format, as long as you give appropriate credit to the original author(s) and the source, provide a link to the Creative Commons licence, and indicate if changes were made. The images or other third party material in this article are included in the article's Creative Commons licence, unless indicated otherwise in a credit line to the material. If material is not included in the article's Creative Commons licence and your intended use is not permitted by statutory regulation or exceeds the permitted use, you will need to obtain permission directly from the copyright holder. To view a copy of this licence, visit http://creativecommons.org/licenses/by/4.0/.

\section{References}

1. Tallet AV, Azria D, Barlesi F, Spano JP, Carpentier AF, Gonçalves A, et al. Neurocognitive function impairment after whole brain radiotherapy for brain metastases: actual assessment. Radiat Oncol. 2012;7:77. https://doi.org/10. 1186/1748-717X-7-77.

2. Khuntia D, Brown P, Li J, et al. Whole-brain radiotherapy in the management of brain metastasis. J Clin Oncol. 2006;24:1295-304.

3. Patchell RA, Tibbs PA, Walsh JW, et al. A randomized trial of surgery in the treatment of single metastases to the brain. NEngl J Med. 1990;322:494-500.

4. Shaw E, Scott C, Souhami L, et al. Single dose radiosurgical treatment of recurrent previously irradiated primary brain tumors and brain metastases: final report of RTOG protocol 90-05. Int J Radiat Oncol Biol Phys. 2000;47:291-8.
5. Andrews DW, Scott CB, Sperduto PW, et al. Whole brain radiation therapy with or without stereotactic radiosurgery boost for patients with one to three brain metastases: phase III results of the RTOG 9508 randomised trial. Lancet. 2004;363:1665-72.

6. Aoyama H, Shirato H, Tago M, et al. Stereotactic radiosurgery plus whole-brain radiation therapy vs stereotactic radiosurgery alone for treatment of brain metastases: a randomized controlled trial. JAMA. 2006;295:2483-91.

7. Soffietti R, Abacioglu U, Baumert B, et al. Diagnosis and treatment of brain metastases from solid tumors: guidelines from the European Association of Neuro-Oncology (EANO). Neuro Oncol. 2017 Feb 1;19(2):162-74.

8. Li J, Bentzen SM, Renschler M, et al. Regression after wholebrain radiation therapy for brain metastases correlates with survival and improved neurocognitive function. J Clin Oncol. 2007;25(10):1260-6. https://doi.org/10.1200/JCO. 2006.09.2536.

9. NayakL, LeeEQ, Wen PY.Epidemiology of brain metastases. Curr Oncol Rep. 2012;14(1):48-54.

10. Barnholtz-Sloan JS, Sloan AE, Davis FG, Vigneau FD, Lai P, Sawaya RE. Incidence proportions of brain metastases in patients diagnosed (1973 to 2001) in the metropolitan Detroit cancer surveillance system. J Clin Oncol. 2004;22(14):2865-72.

11. Berghoff AS, Schur S, Füreder LM, et al. Descriptive statistical analysis of a real life cohort of 2419 patients with brain metastases of solid cancers. ESMO Open. 2016;1(2):e24.

12. Sperduto PW, Berkey B, Gaspar LE, Mehta M, Curran W. A new prognostic index and comparison to three other indices for patients with brain metastases: an analysis of 1,960 patients in the RTOG database. Int J Radiat Oncol Biol Phys. 2008;70(2):510-4.

13. Lauko A, Rauf Y, Ahluwalia MS. Medical management of brain metastases. Neuro OncolAdv. 2020;2(1):1-14.

14. Gondi V, Deshmukh S, Brown PD, et al. NRG oncology CC001: a phase III trial of hippocampal avoidance (HA) in addition to whole-brain radiotherapy (WBRT) plus memantine to preserve neurocognitive function (NCF) in patients with brain metastases (BM). J Clin Oncol. 2019;37(15_suppl):2009-2009. https://doi.org/10.1200/ JCO.2019.37.15_suppl.2009.

15. Gondi V, Deshmukh S, Brown PD, et al. Preservation of neurocognitive function with conformal avoidance of the hippocampus during wholebrain radiotherapy for brain metastases: preliminary results of phase III trial NRG Oncology CC001 [Abstract]. 2018 Annual Meeting ASTRO Abstract LBA9. 2018.

16. Takahashi T, Yamanaka T, Seto T, et al. Prophylactic cranial irradiation versus observation in patients with extensivedisease small-cell lung cancer: a multicentre, randomised, open-label, phase 3 trial. Lancet Oncol. 2017;18(5):663-71.

17. Le Rhun E, Weller M, Brandsma D, Van den Bent M, de Azambuja E, Henriksson R, et al. EANO-ESMO clinical practice guidelines for diagnosis, treatment and followup of patients with leptomeningeal metastasis from solid tumours. Ann Oncol. 2017;28:iv84-iv99.

18. El Shafie RA, Böhm K, Weber D, et al. Palliative radiotherapy for leptomeningeal carcinomatosis-analysis of outcome, prognostic factors, and symptom response. Front Oncol. 2019;8:641.

19. Mulvenna P, Nankivell M, Barton R, et al. Dexamethasone and supportive care with or without whole brain radiotherapy in treating patients with non-small cell lung cancer with brain metastases unsuitable for resection or stereotac- 
tic radiotherapy (QUARTZ): results from a phase 3, noninferiority, randomised trial. Lancet. 2016;388:2004-14.

20. PercyAK, ElvebackLR, Okazaki H, Kurland LT. Neoplasms of the central nervous system. Epidemiologic considerations. Neurology. 1972;22(1):40-8.

21. Tsukada Y, Fouad A, Pickren JW, Lane WW. Central nervous system metastasis from breast carcinoma. Autopsy study. Cancer. 1983;52(12):2349-54.

22. Aupérin A, Arriagada R, Pignon JP, et al. Prophylactic cranial irradiation for patients with small-cell lung cancer in complete remission. Prophylactic cranial irradiation overview collaborative group. NEngl J Med. 1999;341(7):476-84.

23. Gaspar L, Scott C, Rotman M, Asbell S, Phillips T, Wasserman $\mathrm{T}$, et al. Recursive partitioning analysis (RPA) of prognostic factors in three Radiation Therapy Oncology Group (RTOG) brain metastases trials. Int J Radiat Oncol Biol Phys. 1997;37:745-51.

24. Radiation Therapy Oncology Group (RTOG), Brown PD, Pugh S, Laack NN, et al. Memantine for the prevention of cognitive dysfunction in patients receiving whole-brain radiotherapy: a randomized, doubleblind, placebo-controlled trial. Neuro Oncol. 2013;15(10):1429-37.

25. Tsao MN, Lloyd N, Wong RK, etal. Whole brain radiotherapy for the treatment of newly diagnosed multiple brain metastases. Cochrane Database Syst Rev. 2012; https://doi.org/ 10.1002/14651858.CD003869.pub3.

Publisher's Note Springer Nature remains neutral with regard to jurisdictional claims in published maps and institutional affiliations.

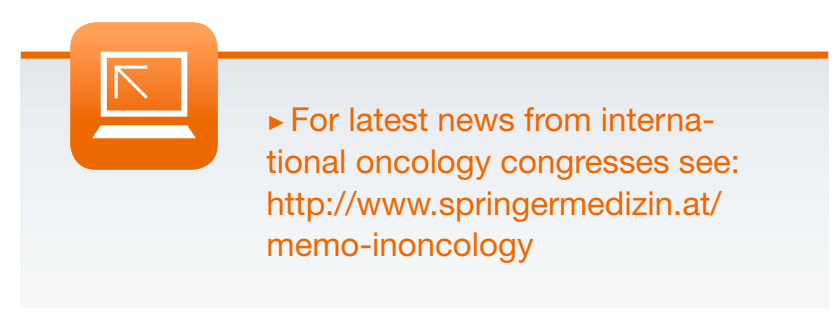

